\title{
UCRL-CONF-155435
}

LAWRENCE LIVERMORE NATIONAL LABORATORY

\section{Assembling and installing LRUs for NIF}

R. E. Bonanno

December 31, 2003

SPIE Photonics West 2004, San Jose, CA, January 24-29, 2004 
This document was prepared as an account of work sponsored by an agency of the United States Government. Neither the United States Government nor the University of California nor any of their employees, makes any warranty, express or implied, or assumes any legal liability or responsibility for the accuracy, completeness, or usefulness of any information, apparatus, product, or process disclosed, or represents that its use would not infringe privately owned rights. Reference herein to any specific commercial product, process, or service by trade name, trademark, manufacturer, or otherwise, does not necessarily constitute or imply its endorsement, recommendation, or favoring by the United States Government or the University of California. The views and opinions of authors expressed herein do not necessarily state or reflect those of the United States Government or the University of California, and shall not be used for advertising or product endorsement purposes. 


\author{
Assembling and Installing LRUs for NIF \\ R. E. Bonanno \\ University of California \\ Lawrence Livermore National Laboratory, 7000 East Avenue, L-466, Livermore, CA 94550
}

\begin{abstract}
Within the 192 National Ignition Facility (NIF) beamlines, there are over 7000 large $(40 \times 40 \mathrm{~cm})$ optical components, including laser glass, mirrors, lenses, and polarizers. These optics are held in large opto-mechanical assemblies called linereplaceable units (LRUs). Each LRU has strict specifications with respect to cleanliness, alignment, and wavefront so that once activated, each NIF beamline will meet its performance requirements. NIF LRUs are assembled, tested, and refurbished in on-site cleanroom facilities. The assembled LRUs weigh up to 1800 kilograms, and are about the size of a phone booth. They are transported in portable clean canisters and inserted into the NIF beampath using robotic transporters. This plug and play design allows LRUs to be easily removed from the beampath for maintenance or upgrades. Commissioning of the first NIF quad, an activity known as NIF Early Light (NEL), has validated LRU designs and architecture, as well as demonstrated that LRUs can be assembled and installed as designed. Furthermore, it has served to develop key processes and tools forming the foundation for NIF s long-term LRU production and maintenance strategy. As we look forward to building out the rest of NIF, the challenge lies in scaling up the production rate while maintaining quality, implementing process improvements, and fully leveraging the learning and experience gained from NEL. This paper provides an overview of the facilities, equipment and processes used to assemble and install LRUs in NIF.
\end{abstract}

\title{
1. INTRODUCTION
}

The National Ignition Facility (NIF) is the world s largest optical and opto-mechanical system. ${ }^{1}$ Each of NIF s 192 individual laser beams can produce up to about 20,000 joules of 1.06 micron laser light in $40 \times 40 \mathrm{~cm}$ square beams. NIF s 192 laser beams contain a variety of precision cleaned and aligned small and large optics located throughout the stadiumsized facility (see Figure 1). NIF s laser beam enclosures, referred to as the beampath infrastructure system (BIS), are required to maintain stringent cleanliness levels on all optical systems to ensure that the high-energy laser light does not interact with surface contaminants on optics that could lead to degraded performance or optics damage over time.

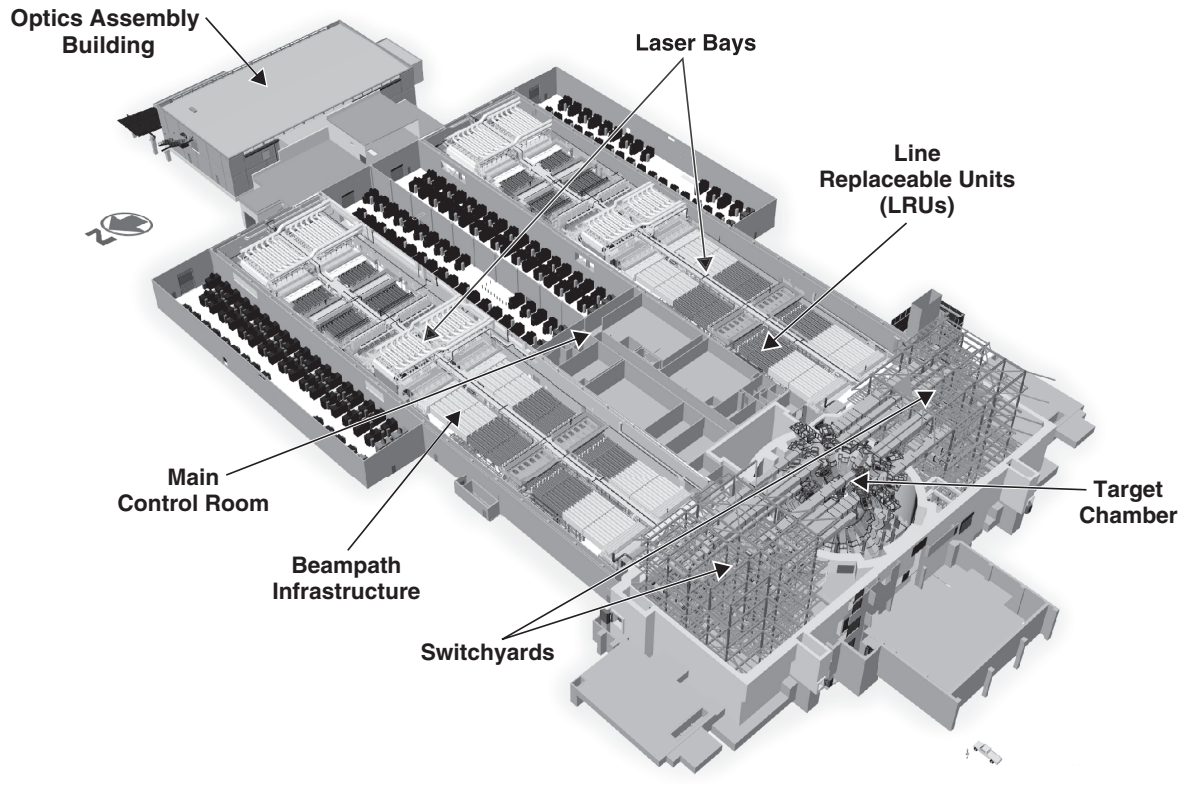

Fig. 1. This computergenerated model of the National Ignition Facility reveals the beampath infrastructure - the precision cleaned and aligned exoskeleton that encloses all laser beam optical and mechanical components within each of the facility s major areas: the laser bays, the switch-yard, and the target area. 
NIF s major laser optical and optomechanical components are built into modular assemblies called linereplaceable units or LRUs. LRUs typically contain up to four beams worth of optics, for example, mirrors, lenses, laser glass amplifier slabs, and polarizers, as well as actuators and kinematic mounts.

Other LRUs contain flashlamps and spatial filter pinhole assemblies.

Figure 2 shows a schematic layout of one NIF laser beam along with the LRUs that are used in different sections of the beampath. LRUs are precision cleaned and assembled in dedicated cleanroom facilities in the Optics Assembly Building (OAB) located adjacent to the NIF Laser building. Special portable cleanrooms, called canisters, are carried

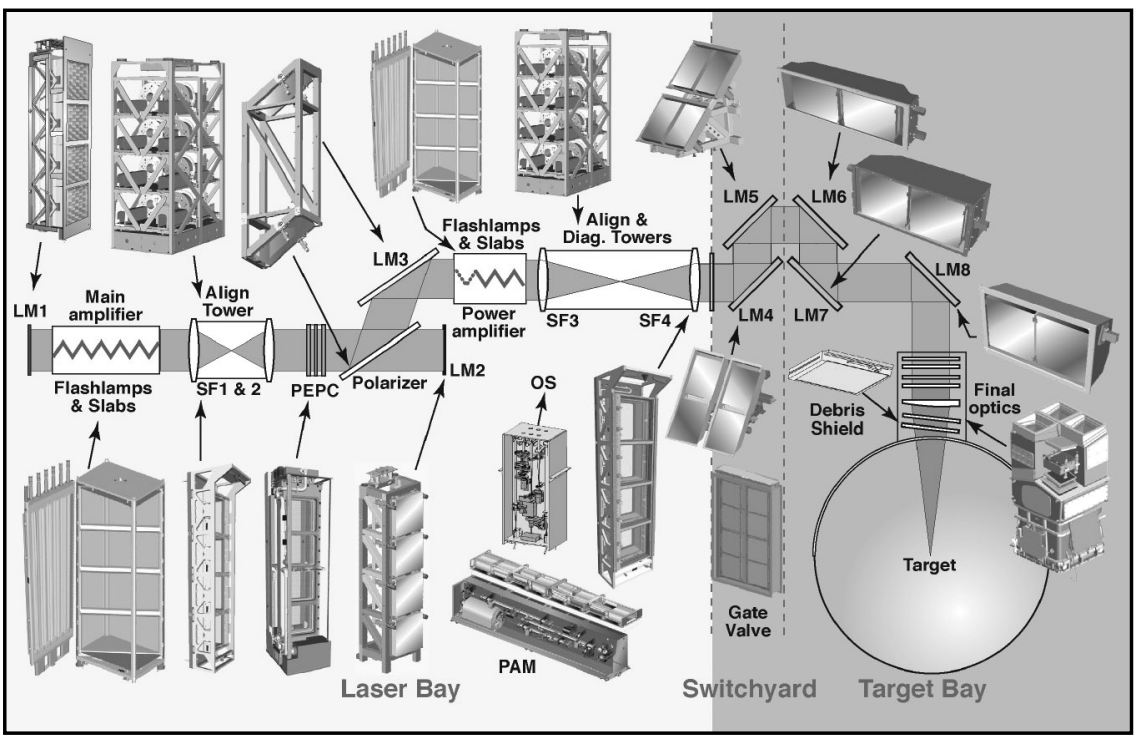

Fig. 2. LRUs are utilized throughout the NIF laser system. This figure shows a single NIF beamline indicating where different LRUs are located in the line. Many LRUs, for example, laser glass amplifier slab cassettes, contain optics stacked four high for four NIF laser beams. by autonomously-guided vehicles (AGVs) and are used to transport LRUs between the OAB and the laser bays. The canisters are designed to allow the insertion and removal of LRUs in NIF s beampath, while preserving the clean environment inside the beampath.

LRU assembly and installation began in 2002, but the groundwork for building the laser was put in place beginning in the mid-1990s. Figure 3 shows the sequence of major project elements that were required before installation of LRUs into NIF could begin. This work involved building and preparing the beampath infrastructure; installing the electrical and other utilities required to operate the LRUs; procurement and fabrication of the LRU hardware; and the special equipment necessary to assemble, test, and install the LRUs. The first 127 LRUs have now been installed in NIF in

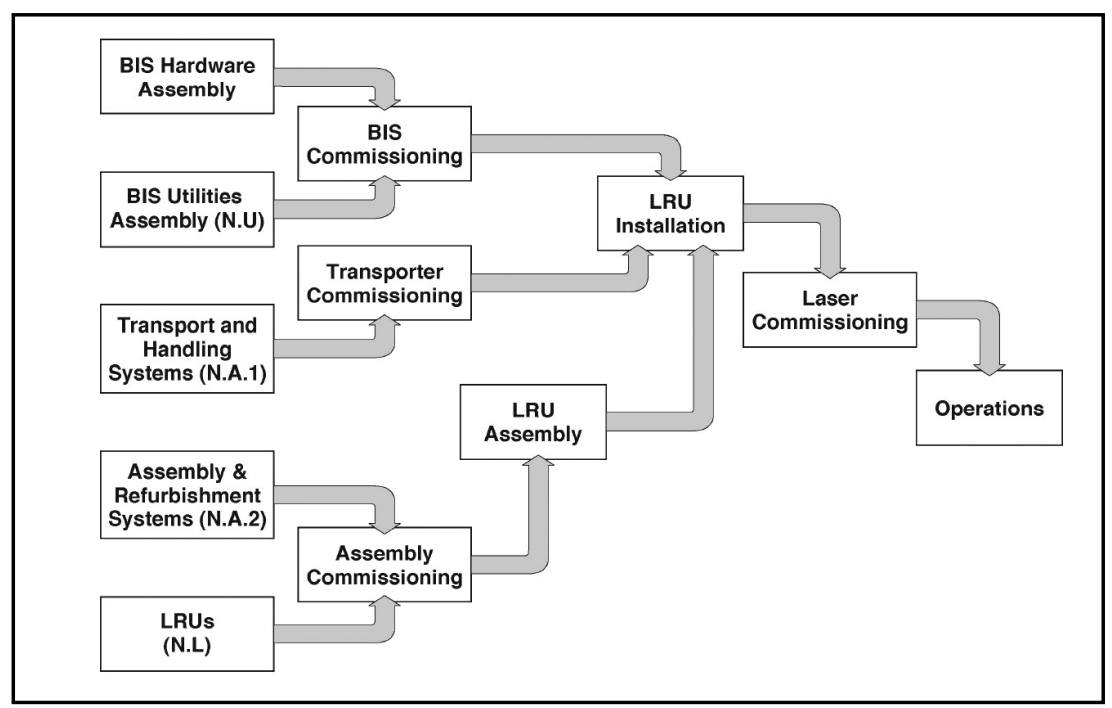
support of commissioning the first four NIF laser beams. Commissioning of these four beams, also referred to as a quad, was an activity known as NIF Early Light (NEL). Experience with the assembly, installation, and refurbishment of NIF LRUs indicates that classic production process learning theory applies to the assembly of NIF LRUs, and that transport and handling of LRUs during insertion and removal is successfully maintaining NIF s cleanliness and laser operating requirements.

Fig. 3. LRU assembly and installation required other major laser systems such as the beampath infrastructure system (BIS) and transport and handling systems to be installed and commissioned. 


\section{LRU ASSEMBLY AND INSTALLATION FACILITIES AND EQUIPMENT}

LRUs are assembled, aligned, and tested prior to installation in the NIF beampath. The NIF Project maintains a number of dedicated cleanroom facilities at Lawrence Livermore National Laboratory (LLNL), and at off-site vendor facilities. Each of these facilities contains a wide variety of specialized equipment used for optics processing, LRU assembly and LRU installation. The requirements for these systems presented a number of unique engineering challenges. These systems must manipulate and transport optics, LRU subassemblies and fully assembled LRUs, which are large and can weigh up to $1800 \mathrm{~kg}$. At the same time, these operations must be performed with high precision in order to meet tight alignment tolerances, must be accomplished without introducing contaminants or particulates that would affect the cleanliness level of the LRU or the beampath, and must not damage high-value optics and other precision components. They must also be highly reliable and maintainable in order to meet NIF s operational goals. Industrial equipment for handling large and heavy objects is not typically designed to operate in a cleanroom, and conversely, cleanroom-qualified equipment is not typically designed to handle LRU-sized assemblies. The majority of this equipment was designed by LLNL engineers but where ever possible proven industrial methodologies and technologies were incorporated. Prior to assembling LRUs that would actually be used in the operating laser, these systems were extensively exercised and qualified using surrogate hardware and optics. The NEL activity has allowed us to fully qualify these systems for operation, and has demonstrated that NIF requirements for these systems have been met.

Optics and some component-level mechanical assemblies are cleaned, coated, and assembled in the 400 -square-meter Class 100 Optics Processing Laboratory (OPL). The OPL consists of two distinct and physically separated areas. One area is dedicated to processing fused silica optics and laser glass. It contains aqueous, ultrasonic cleaning systems used for precision cleaning the optics, sol-gel coating systems for applying anti-reflection (AR) coatings to lenses and windows, an ammonia/water vapor curing chamber which hardens and increases the robustness of the AR coatings, and several workcenters for integrating certain optics into LRU sub-assemblies. The other major area in the OPL is used for cleaning and coating potassium dihydrogen phosphate (KDP) crystals which are used in several NIF LRUs. This area is physically separated from the area where water-based processes are performed due to the deleterious effects of water vapor on KDP substrates. Optics are moved from station to station in the OPL using handling and manipulating equipment designed to virtually eliminate human contact with the optics and to prevent chipping and other types of optic damage due to handling. Several different types of metrology and inspection systems are used during optic processing to verify that each optic meets requirements. These include a large-aperture photometer and ellipsometer for measuring reflectance of AR coated optics, a cleanliness verification system for measuring surface cleanliness, and a $24 "$ interferometer for measuring the wavefront of certain optic sub-assemblies.

Optics and optic sub-assemblies are then transported to the OAB co-located at the NIF site where they are integrated with the mechanical hardware and other components which comprise each LRU. The OAB consists of a 400 -squaremeter Class 10,000 cleanroom with large ultrasonic systems for precision cleaning LRU mechanical hardware, and a 400-square-meter Class 100 cleanroom containing dedicated workcenters for assembling the different types of LRUs. Although each workcenter is unique to the type of LRU assembled there, each generally consists of a rotating platform which supports the LRU frame through the assembly process at various angles and positions, and an optic insertion device which picks-up and precisely positions each optic in the LRU frame.

In order to meet NIF goals for laser shot rate and availability we must ensure that LRUs are built correctly the first time and will meet performance requirements once installed. Before an LRU is installed into the beampath it undergoes a rigorous set of inspections and verifications to ensure that it will meet requirements for wavefront, alignment, cleanliness and functionality of actuators and other electronic components. The OAB contains several large-scale, LLNL-designed verification systems. The LRU alignment verification system (LAVS) is used to verify 

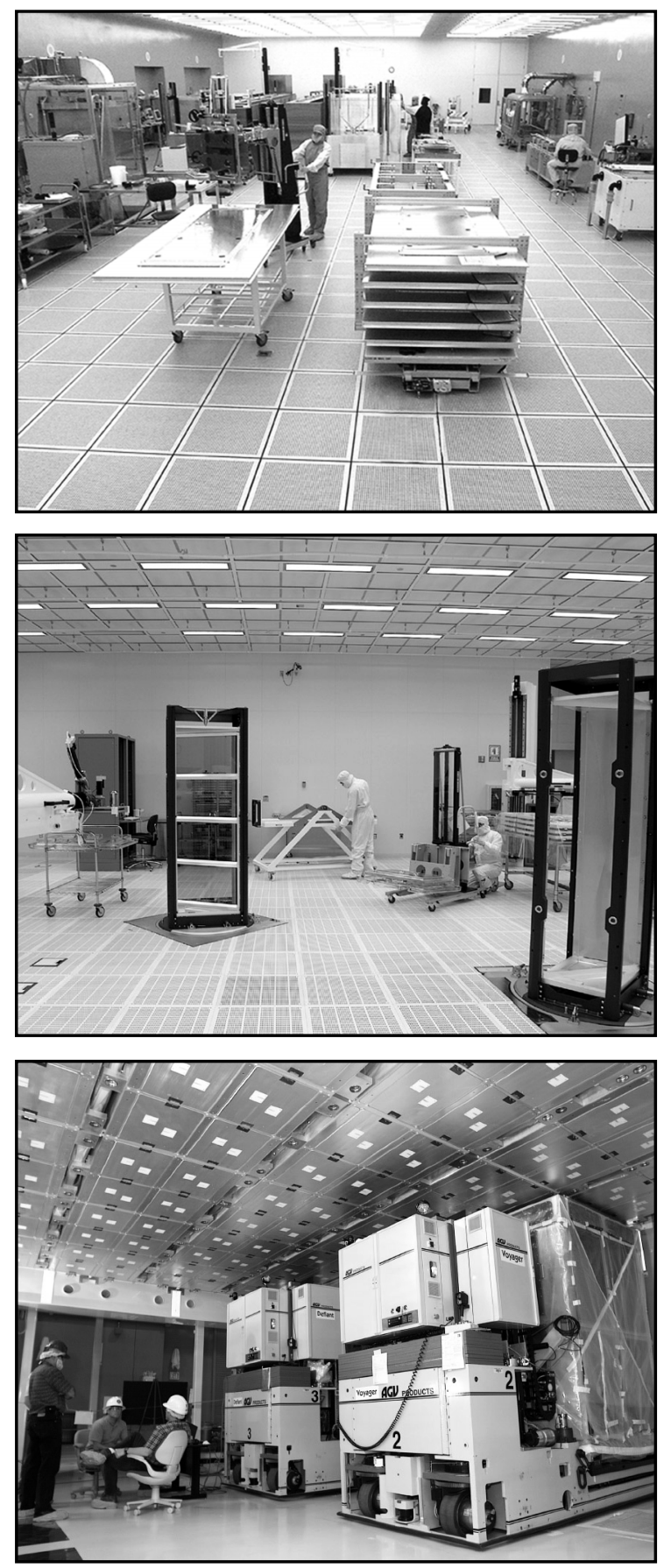

Fig. 4. Photograph at top shows the Optics Processing Laboratory (OPL). Flashlamp windows are stacked in the foreground, and a technician is moving a laser amplifier glass slab. The laser glass is assembled into a four-slab cassette in the OAB shown in the middle photograph. Laser glass cassettes are transported into the laser bay and inserted into the beampath using special portable cleanrooms carried by autonomously-guided vehicles (AGVs), shown in the bottom photograph. that each optic is correctly positioned in the LRU frame. LAVS consists of an ultra-stable steel structure with docking locations for the different types of LRUs. In the center of this structure is a robotic arm which can accommodate video, ultrasonic and auto-collimator based metrology tools. The optics are precisely scanned in three dimensions to ensure their $\mathrm{x}, \mathrm{y}$ and $\mathrm{z}$ positions relative to the LRU frame are within tolerance, and that they are oriented at the correct pre-alignment angle. The position tolerances vary by LRU but are typically $-1.0 \mathrm{~mm}$ in the $\mathrm{x}, \mathrm{y}$, and $\mathrm{z}$ directions, and $-1.0 \mathrm{mrad}$ in angular tilt. Pre-aligning the position of the optics prior to installation decreases the time required to perform on-line alignment and activation of the laser. The wavefront verification system (WAVES) is a Hartmann sensor-based diagnostic that measures the wavefront distortion across the full aperture of a large optic in an assembled LRU. This system verifies that mount-induced wavefront distortion is within tolerance. The tolerance also varies by LRU but ranges from $0.10-0.25$ waves (peak-to-valley).

Once assembled and tested the LRU is ready for transport and installation into NIF. The transport and installation system consists of two major sub-systems: canisters and AGVs. Canisters are portable cleanrooms which carry the LRU during transport. Inside the canisters are mechanisms which dock to the NIF beampath, and precisely lift and set the LRU on kinematic mounts inside the beampath. There are three types of canisters that accommodate the different LRUs installed in the NIF laser bays. AGVs carry the canisters and move between the OAB and the NIF laser bays. AGVs are robotically-controlled using a commercial traffic management system and are programmed to pick-up LRUs from the OAB and to precisely position the canister at the specified installation location. This process is reversed when a LRU is removed for servicing. In addition to maintaining the cleanliness level of the LRU these systems must also minimize vibration and shock to the assembly.

These facilities and some transport and installation systems are shown in Figure 4. 


\section{LRU PRODUCTION}

Over 4,000 LRUs are required to be produced and installed in NIF between now and Project completion in 2008. The process of fabricating, assembling, and tracking the hundreds of thousands of parts needed for the LRUs is managed through a rigorous system of work orders and software-based tracking tools. A flow diagram of NIF s LRU production management and control process is depicted in Figure 5. It illustrates the flow of parts and information as well as the role of each Project organization that participates in building and installing LRUs in NIF. The production cycle is initiated by the LRU demand schedule which specifies the date each LRU must be available for installation. The LRU demand schedule is driven by the Project s master schedule which inter-relates all elements required for laser commissioning.

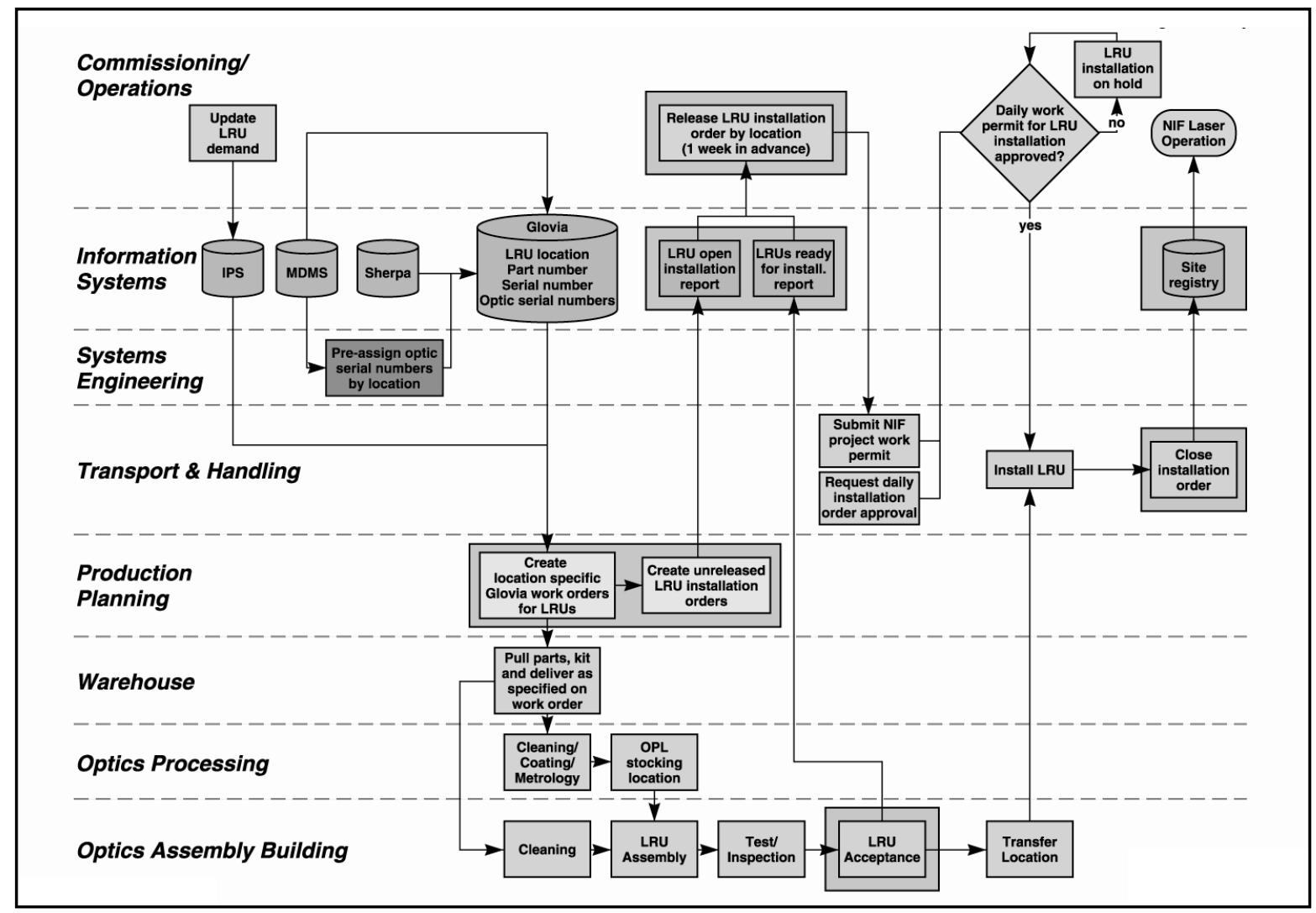

Fig. 5. Process flow diagram for LRU production management and control.

A key part of managing LRU production is the use of software tools designed to allow tracking of parts throughout the process of assembly. Glovia, a commercially available enterprise resource planning (ERP) application, has been adapted for use at NIF. The first step in the LRU production cycle is to initiate a work order in the Glovia system which will track bar-coded parts and assemblies through all the processing, assembly and verification steps. In addition, all parts assembled into an LRU are archived by a configuration management system which also retains test and installation data for future reference.

To further illustrate the NIF LRU production cycle, a description is provided in Figure 6 of the process for assembling and installing the plasma electrode Pockels cell (PEPC). The PEPC is a four-aperture optical switch that confines laser light in the main amplifier section of NIF for multiple passes using polarization rotation and a separate polarizing optics assembly. ${ }^{2}$ 
(a)
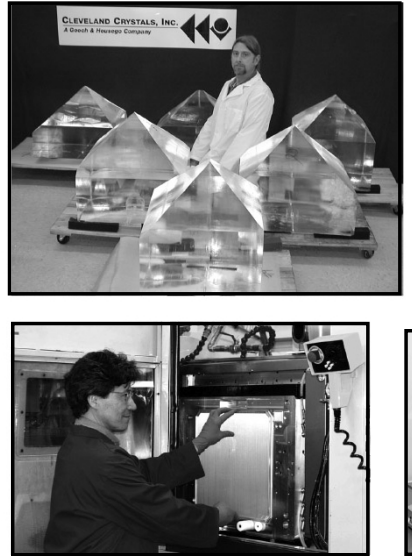

Potassium dihydrogen phosphate (KDP) crystals are grown, machined, finished, and inspected at the vendor prior to shipping to NIF.

(c)

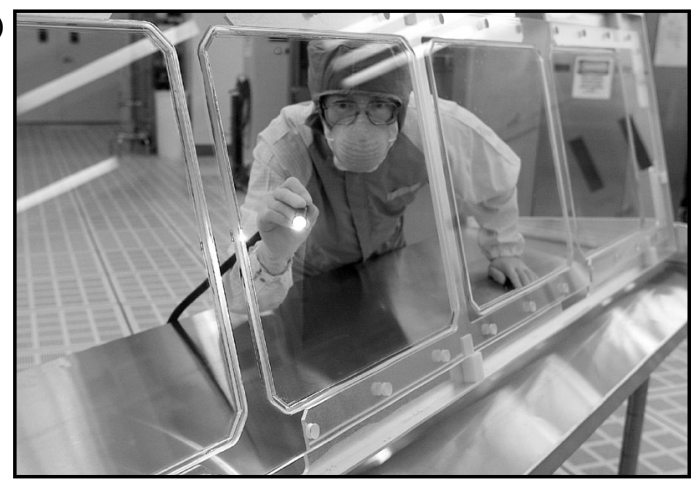

Finished crystal plates are assembled into the PEPC mid-plane frame and inspected in the OPL.

(e)

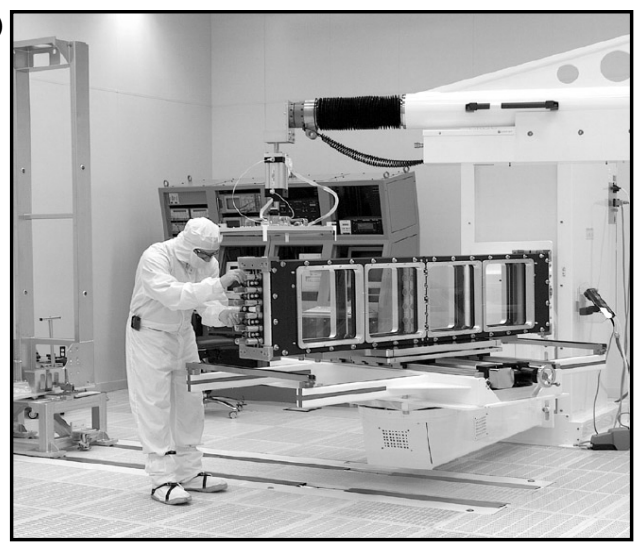

Work continues on the PEPC with final electrical and gas connections being installed. (b)

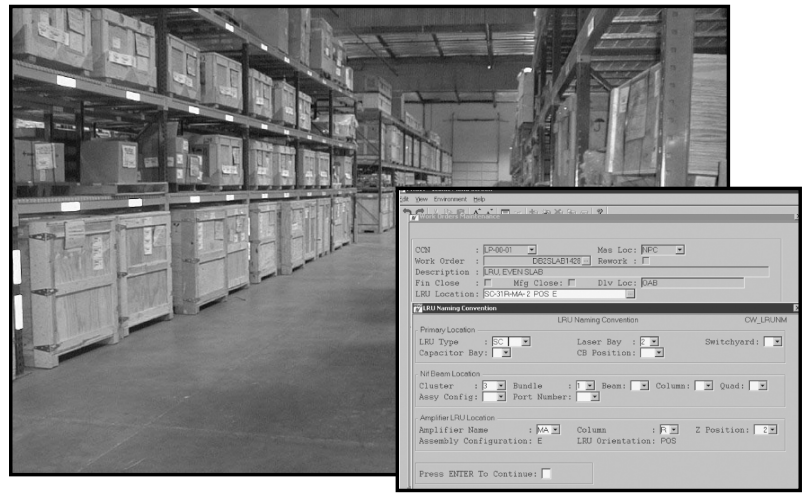

Optics and mechanical parts are received and warehoused until needed.

To begin the process, a work order is opened which will track the work at each location as the parts and optics are assembled and installed.

(d)

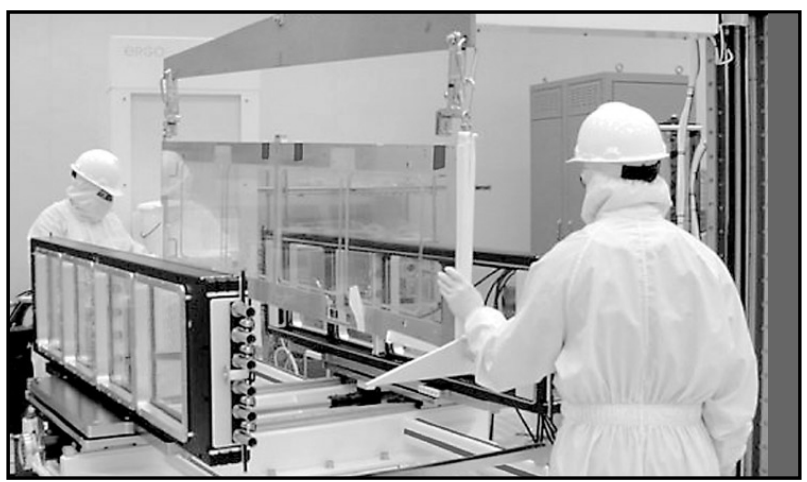

PEPC mid-planes are placed into frame assemblies using a dedicated workstation in the $\mathrm{OAB}$. (f)

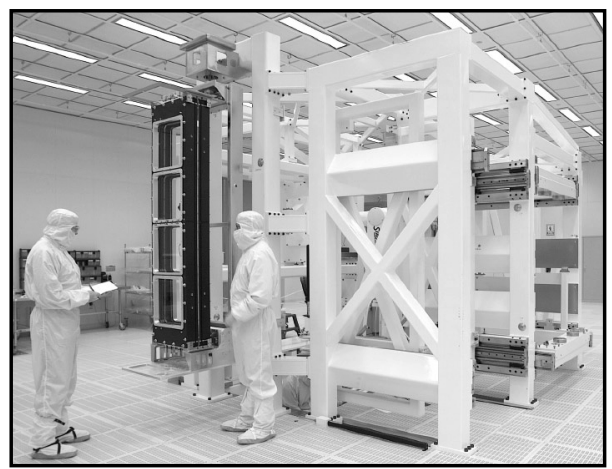

Alignment and electrical performance of the assembled PEPC are verified at test stations in the OAB prior to acceptance of the LRU for installation.

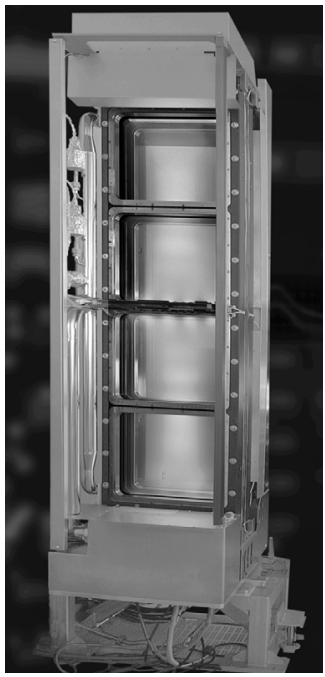

Fig. 6. Sequence of PEPC assembly and installation process (continued on next page). 
(g)

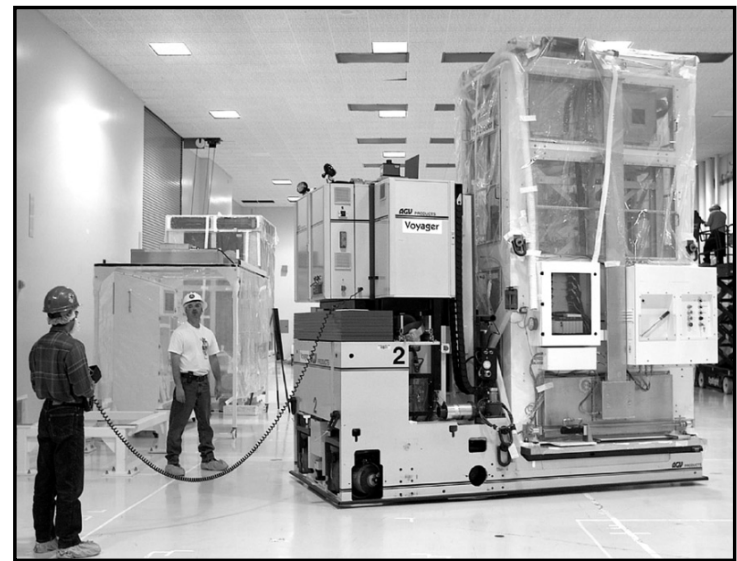

Finally, the LRU is loaded into a canister and transported to the specified beampath location where it will be installed. (h)

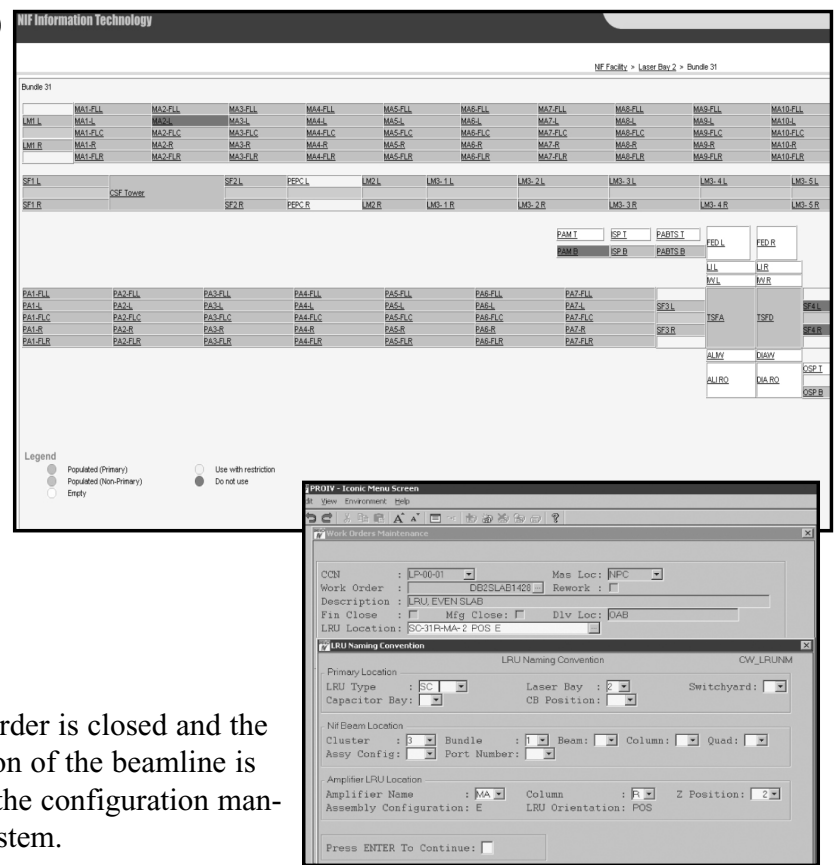

Fig. 6. Sequence of PEPC assembly and installation process (continued).

\section{LRU PROCESS VALIDATION}

In the process of designing NIF many new systems were required for assembling, installing, and maintaining NIF LRUs. Over the past few years as these systems reached design maturity, early prototypes and production process studies have been superseded by final production systems and validated models. The understanding of our production processes is extremely important as we enter into the next phase of NIF s construction involving assembly and installation of over 4,000 remaining LRUs through Project completion in 2008. The next phase of building NIF becomes more complex as the laser beamlines already commissioned will continue to operate concurrently with LRU installation and activation of the remaining beamlines.

Process studies and improvements based on lessons learned installing the first four beams worth of LRU hardware are being performed and are helping plan future LRU production. Figure 7 shows an example of the learning curve associated with the production of flashlamp windows in the OPL. The curve follows classical learning theory for efficiency gained from performing repetitive tasks. Using a commercial simulation and scheduling package, a discrete-event simulation of NIF LRU production has been developed. Actual cycle time and learning curve data from these early production activities has been used to validate the simulation which fully models the LRU production and installation process for all 192 beamlines. The simulation is a useful tool for identifying and mitigating potential bottlenecks, resource conflicts and scheduling issues. It is also being used by Project managers to validate goforward strategies, costs and schedules to ensure that remaining Project milestones will be met.

Another important aspect of this work has been the validation of clean assembly, insertion, and removal of LRUs while maintaining strict cleanliness standards on NIF optical components and within the interior of the beampath. Early insertion and removal tests conducted, in some cases using surrogate parts, first established that clean conditions could be maintained during transport and handling operations. More recent experience with actual NIF LRUs continues to show that cleanliness levels are maintainable on a routine basis. 


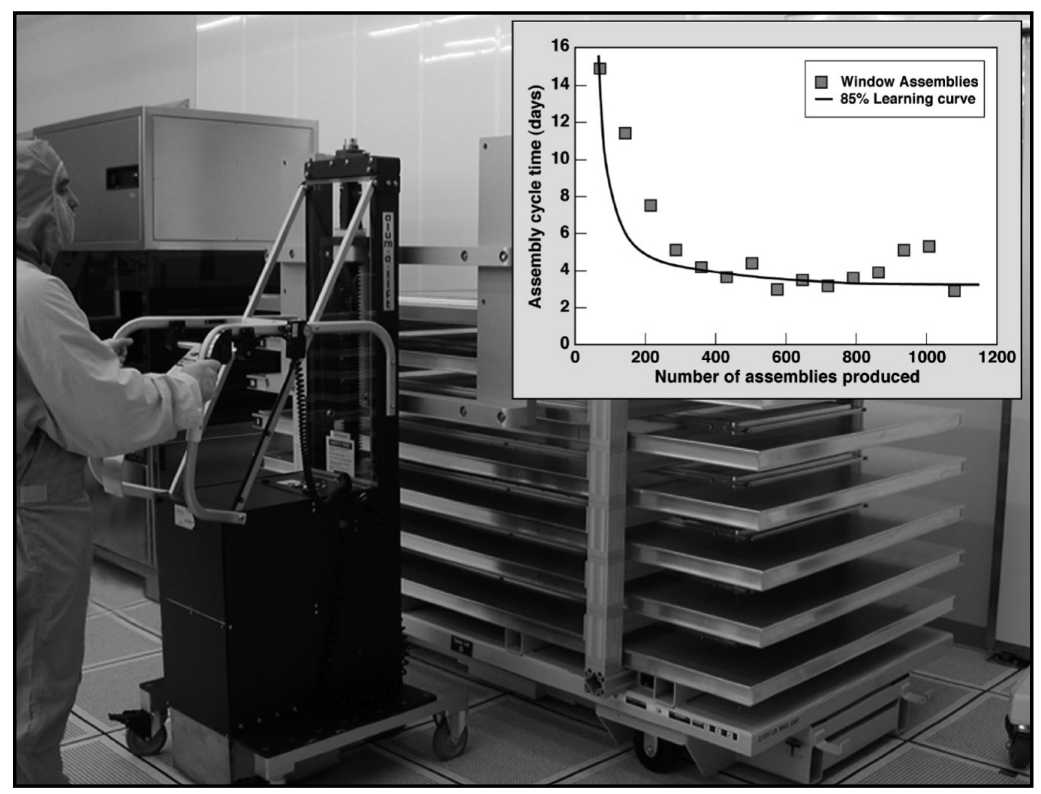

Fig. 7. As technicians gained more experience preparing and assembling flashlamp window assemblies in the OPL, the cycle time improved according to an $85 \%$ learning curve.

\section{CONCLUSIONS AND PATH FORWARD}

The Assembly, Installation, and Refurbishment group at NIF is preparing to increase its activities starting in late FY 2004 as the next beams of NIF begin to be installed. Over the following four years an intense period of activity will take place with LRU production averaging five LRUs per day in order to meet NIF s completion milestones (see Figure 8). The experience gained during early engineering prototyping, followed by the assembly and installation of

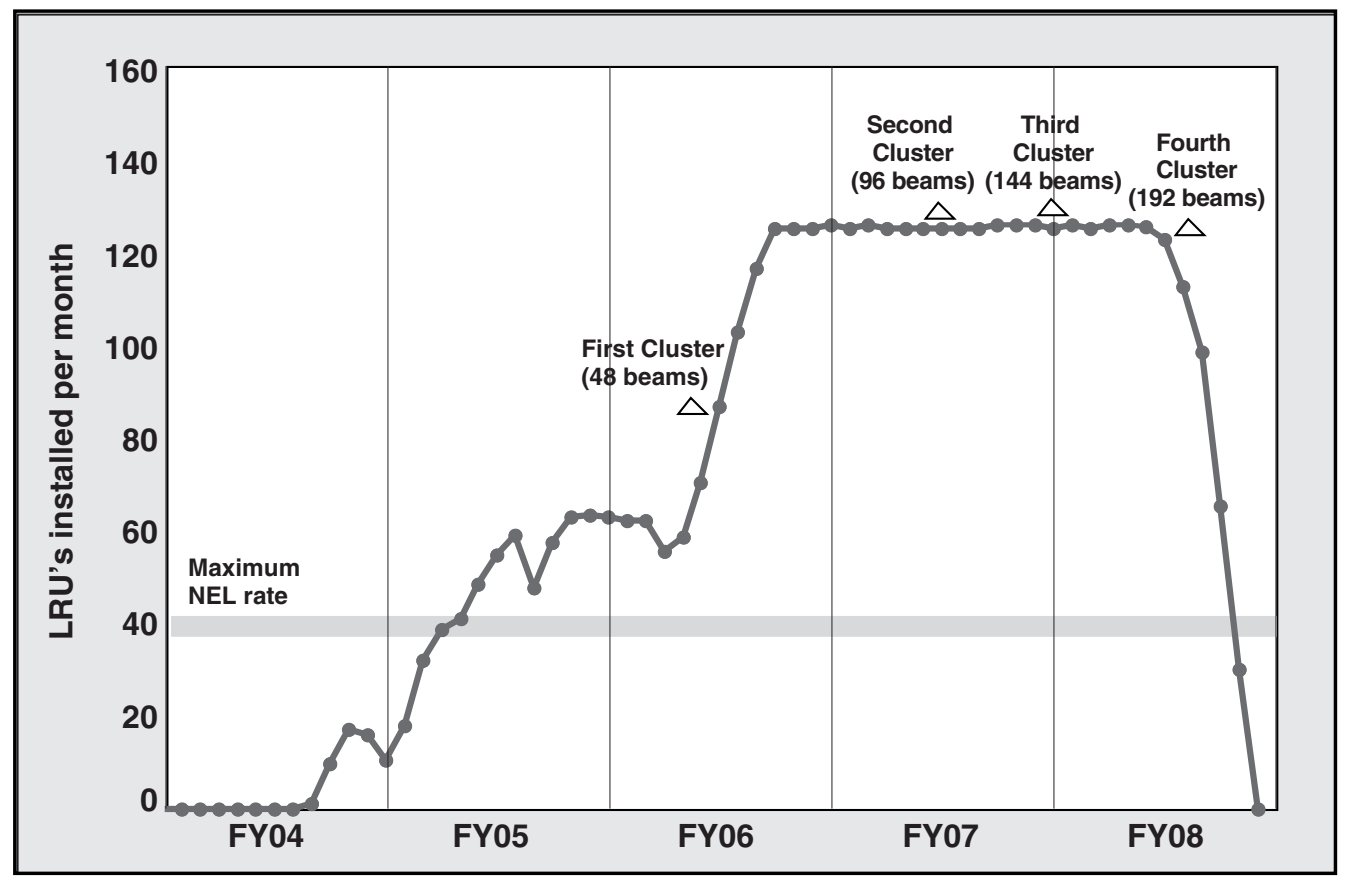

Fig. 8. Monthly production and installation rate for the completion of NIF s 192 beamlines. 
over 120 LRUs during the past year, along with the valuable operational experience and training of our high-quality technical staff, will help to ensure that NIF achieves its goals of being the world s largest and most energetic laser system for basic and applied high-energy-density and inertial fusion energy science. ${ }^{3,4}$

\section{ACKNOWLEDGEMENTS}

This work was performed under the auspices of the U.S. Department of Energy by the University of California, Lawrence Livermore National Laboratory under contract No. W-7405-Eng-48.

\section{REFERENCES}

1. E. Moses, et al., The National Ignition Facility: The World s Largest Optics and Laser System, UCRL-JC-151593, SPIE Proc. 2003, Photonics West, January 2003.

2. C. Boley and M. Rhodes, Modeling of Plasma Behavior in a Plasma-Electrode Pockels Cell, IEEE Trans.on Plasma Science, 27, 3, June 1999.

3. J. Lindl, Inertial Confinement Fusion: The Quest for Ignition and Energy Gain Using Indirect Drive, Springer-Verlog, New York, 1998.

4. S. Glenzer, et al., Progress in Large-Scale Length Laser Plasma Interactions, UCRL-CONF-155301, to be published in the Proceedings of the Third International Conference on Inertial Fusion Science and Applications, Monterey, CA, September 2003. 